\title{
Recruitment Source and Behavioral Risk Patterns of HIV-Positive Men Who have Sex with Men
}

\author{
Holly H. Fisher · David W. Purcell · Colleen C. Hoff · \\ Jeffrey T. Parsons · Ann O'Leary
}

Published online: 20 October 2006

(C) Springer Science+Business Media, LLC 2006

\section{Erratum to: AIDS Behav \\ DOI 10.1007/s10461-006-9109-3}

This erratum is concerned with printer errors. On page 554, the first section under "Measures" should be entitled "Demographics, health characteristics, and sexual identity". In addition, on page 555, in the "Demographics, health characteristics, and sexual

identity" section, the correct sexual orientation categories in this measure are "gay/queer/homosexual," "straight/heterosexual," "bisexual," and "none of the above/unsure". In addition, there are formatting errors in the tables that are not the fault of the author. Readers should note these corrections and the Editorial office apologizes for the errors.
The online version of the original article can be found at http://dx.doi.org/10.1007/s10461-006-9109-3.

H. H. Fisher $(\varangle) \cdot$ D. W. Purcell · A. O'Leary

Prevention Research Branch, Division of HIV/AIDS

Prevention, National Center for HIV, STD, and TB

Prevention, Centers for Disease Control and Prevention,

1600 Clifton Road, MS E-37, Atlanta, GA 30333, USA

e-mail: hkh3@cdc.gov

C. C. Hoff

Center for AIDS Prevention Studies, University of

California, San Francisco, CA, USA

J. T. Parsons

Hunter College and the Graduate Center of the City

University of New York, Center for HIV/AIDS Educational

Studies and Training, New York, USA 